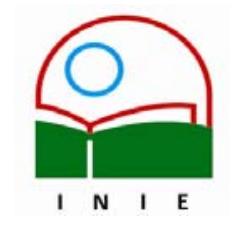

Universidad de Costa Rica

Facultad de Educación

Instituto de Investigación en Educación

ACTUALIDADES INVESTIGATIVAS EN EDUCACION

\title{
EDUCAR EN COMUNIDAD: IMPLICA MUCHO MÁS QUE BUENA VOLUNTAD; ES UN ESPACIO PARA CELEBRAR LA DIVERSIDAD
}

\author{
EDUCATING IN THE COMMUNITY: IMPLIES MUCH MORE THAN GOOD WILL; \\ IT IS A SPACE TO CELEBRATE DIVERSITY
}

Teresita Cordero Cordero ${ }^{1}$

\begin{abstract}
Resumen: El presente artículo plantea la discusión sobre una serie de acciones que se dan en el marco institucional e investigativo, relacionados con los estudios sobre la crianza infantil y el programa de los Hogares Comunitarios en Costa Rica. Estos abordajes no cumplen con una perspectiva integradora y menos involucran procesos de desarrollo humano sostenible. Para ello se apela al cambio de prácticas y de enfoques conceptuales. Se señalan limitaciones sobre las prácticas institucionales, así como se numera una serie de posibles acciones alternativas. Es un estudio que cuestiona, no las intenciones, pero sí las prácticas institucionalizadas, lo que nos lleva a impulsar la creación de alternativas críticas, tarea que demanda de la acción conjunta. Por tal razón, el tema está abierto al debate.
\end{abstract}

\section{Palabras clave: CRIANZA INFANTIL/ EDUCACIÓN PREESCOLAR NO FORMAL/TRABAJO COMUNITARIO/}

\begin{abstract}
The present article expounds the discussion of a series of actions that take place in the institutional and research framework related to studies on child rearing and the Community Homes Program in Costa Rica. These approaches do not posses an integrative perspective and do not involve sustainable human development processes. To address these problems, this work proposes a change in practices and of conceptual focus. It therefore points out limitations in the institutional practices and enumerates a series of possible alternative suggestions. This work questions not the intensions but rather the institutionalized practices, which requires the creation of critical alternatives, a task that demands joint actions. This work is open to debate and discussion.
\end{abstract}

Key words: CHILD REARING/ NON-FORMAL PRESCHOOL EDUCATION/ COMMUNITY WORK

\footnotetext{
1 Doctora en Educación del Programa Latinoamericano de Educación, Master en Psicología ambos títulos de la Universidad de Costa Rica. Desde 1991 es profesora en la Escuela de Psicología de la UCR, ocupa el puesto de subdirectora en la misma Escuela y es investigadora en el Instituto de Investigación en Educación (INIE) de la UCR. Ha ocupado los puestos de coordinadora de Docencia de la Escuela de Psicología, profesora investigadora en el Instituto de Investigaciones Psicológicas (IIP). Se ha desarrollado en el campo de la Psicología Social Comunitaria $y$ actualmente esta estudiando los temas de desarrollo infantil, estudios culturales y educación.
}

Correo electrónico: tcordero@cariari.ucr.ac.cr

Artículo recibido: 6 de enero, 2006

Aprobado: 24 de abril, 2006 


\section{Introducción}

La temática relacionada con la infancia sigue cobrando importancia para las sociedades actuales. Las discusiones y prácticas sobre la atención y cuidado infantil, se convierten en un foco de atención, el cual se evidencia en los estudios realizados desde el siglo pasado, así como, en la conciencia social de la importancia de la niñez y el respeto de sus derechos humanos ${ }^{2}$.

El desarrollo de los abordajes científicos y las prácticas de intervención institucionales, delimitan una serie de conceptos, ideas y acciones para realizar trabajo con la población infantil y sus familias. No obstante, algunas de las acciones institucionales no han podido ser eficaces en sus esfuerzos, dando al traste con los buenos propósitos con los cuales nacen.

El presente trabajo es un ensayo sobre algunas ideas de la autora, producto de la experiencia y revisión de información. El interés es ofrecer un material que sirva para discusión, ya que señala limitaciones sobre prácticas institucionales, así como insiste en presentar una perspectiva conceptual, la cual se sugiere ser considerada a la hora de realizar proyectos educativos o investigativos. Por supuesto, que este material no es un producto final, sino que es más bien un esfuerzo por polemizar sobre dos temas (investigación de crianza infantil y desarrollo de un programa de educación comunitario) relacionados con el desarrollo infantil.

Como tema y problemática se hace referencia a la ciencia como productora de conocimiento y de desconocimiento; afirmación que se sustenta por la exposición de estudios que nos suelen informar muy bien del problema, pero que no siempre tienen impacto social, ni resuelven los problemas que exponen. Esto crea la sensación de que estamos haciendo mucho sin llegar efectivamente a lo que se enuncia.

Los ejemplos que se retoman en este trabajo, corresponden a estudios o reportes de investigación. Uno de ellos es sobre los Hogares Comunitarios en Costa Rica , los cuales se basan en trabajos finales de graducación realizados en la Universidad de Costa Rica, durante los años noventa y que nos indican las limitaciones y algunos aciertos del programa. 
Adicionalmente, se expone la información del programa colombiano, que fue una de las fuentes de inspiración a dicha experiencia.

En el campo de la investigación se retoman trabajos relacionados con el tema de la crianza infantil y, se menciona la importancia de establecer enfoques integradores, en una suerte de avance en el conocimiento.

Se finaliza con un pequeño listado de señalamientos que buscan impulsar la discusión y reflexión crítica sobre las prácticas profesionales y académicas; las cuales, contienen como telón de fondo un compromiso ético impostergable.

\section{Planteamiento general del problema}

Tanto en la psicología ${ }^{3}$ como en la pedagogía, las nuevas tendencias, critican "el carácter universalista y etnocéntrico que ha predominado" (Rosabal, 2004, p. 4). Lo que nos indica, entre otros, la necesidad de tomar en cuenta ejes como son: la protección integral de los derechos humanos; la contextualización y comprensión de las particularidades en que se ven envueltas la realidad socio-cultural de las poblaciones con las que trabajamos.

El carácter de universalidad del conocimiento en el estudio de la niñez, ha estado influido por la ciencia positiva, cuya pretensión ha sido la búsqueda de leyes universales y la consecuente generalización de "modelos" y "patrones" que puedan servir para replicarlos en cualquier lugar. Dicha conceptuación parte de que el desarrollo humano es estático y que puede conocer el o los "patrones" de funcionamiento y la viabilidad de replicarlos en un afán de controlar la vida en sociedad.

Sin embargo, ya es conocido que la constitución humana es dinámica y está en constante transformación, ya que responde a una multiplicidad de situaciones (Ibañez, 1994). Ante

${ }^{2}$ Dos ejemplos concretos son la convención de los Derechos del Niño (1989) ratificada en Costa Rica y el Código de la Niñez y la Adolescencia aprobado el 6 de enero de 1998 (ley 7739).

3 Se contemplan las disciplinas, de las cuales la autora, tiene autoridad para expresar, por su formación, pero, se tiene claro que a lo mejor otras personas, también las podrían contemplar desde otras disciplinas. 
esto, cabe retomar la crítica que esboza Gergen (1996), con respecto al conductismo en las ciencias sociales y en específico en la psicología, porque desde este se han estudiado los patrones de crianza de manera muy simplista, haciendo énfasis en la díada (madre-hijo o hija), limitando el campo de acción y el estudio de una temática tan compleja. Así, la pretensión ha sido conocer los mecanismos, ideas y las conductas, de cómo las madres educan a sus hijos e hijas, con el objetivo influir en dichas "variables" para conseguir cambios en la dinámica social. No obstante, el autor expone que esta condición de inteligibilidad no corresponde con respuestas a las necesidades de la realidad social.

Así en el campo del desarrollo infantil en Costa Rica no encontramos con los estudios de Álvarez; Brenes \& Cabezas; 1991; CELAM \& UNICEF, 1994; MEP \& OEA, 1992 y 1994, los cuales buscaron dar cuenta de los patrones de crianza de la población en general, con la pretensión de conocer de manera uniforme ciertas conductas y variables que diera pistas para su intervención social.

En el caso de Álvarez, Brenes \& Cabezas (1991), el documento nos presenta en su marco conceptual y metodológico, la complejidad de la temática, la cual se relaciona con: la relatividad cultural; los instrumentos de medición; la complejidad del tema y la necesidad de utilizar otras metodologías como la observación sistemática. Además, CELAM y UNICEF (1994), enfatizan que los patrones de crianza son susceptibles a los cambios que sufren las sociedades de manera permanente; pero dichos trabajos no escapan al sesgo positivista reflejado sobre todo por los abordajes metodológicos, omitiendo que:

Metodológicamente es importante implementar diseños lo suficientemente flexibles que puedan adaptarse a realidades diversas y no generar el proceso contrario: forzar las realidades a los métodos. (Baltes y otros, 1988, citado por Rosabal, 2004, p. $5^{4}$ )

Carmiol (2003), por su parte, desde una perspectiva de avance, incorpora en su análisis la condición social de las madres participantes e introduce otras metodologías; Moreno (2002), desde otra perspectiva menciona aspectos de las dinámicas de relación social que son

\footnotetext{
${ }^{4}$ Baltes, P. B; Reese, H:W.; Nesselroade, J.R. (1998) Life-Span Psychology: Introduction to research methods. New Jersey: Lawrence Earlbaum Assoc. Citado por Rosabal (2004) 
influyentes en la crianza infantil ${ }^{5} \mathrm{y}$, ambos indican la necesidad de incorporar otros factores en el abordaje de esta problemática.

Por tales razones, se tiene la necesidad de avanzar en la tarea de incorporar abordajes integradores y novedosos para superar lo que se ha llamado, en este caso, "patrones" de crianza.

El objetivo no es desestimar estudios sobre la crianza infantil, sino exponer la necesidad de buscar un marco amplio, que pueda tomar en consideración metodologías y planteamientos integradores que rompan con una visión lineal y homogénea de la vida social; que considere el contexto en el que se desarrolla la vida infantil; que reconozca la riqueza de lo diferente; que estudie no solo a la madre y el hijo, sino que incluya a otros sujetos que son parte constituyente y, que se consideren los procesos culturales ${ }^{6}$ en la formación humana.

A manera de ejemplo, recordemos cómo los niños y las niñas de las zonas rurales establecen una relación distinta con los elementos de la naturaleza, en comparación con infantes que viven en la ciudad. En este sentido, las y los profesionales, en ocasiones, perdemos de vista lo particular, aunque lo tengamos enfrente de nuestros ojos y caemos en la trampa de tratar de ajustar la realidad a nuestras preconcepciones aprendidas, descalificando o desvalorizando otras formas alternativas de enfrentar los procesos educativos. Tal como lo exponía, la compañera Hellen Valverde (2005), en una presentación pública, hay que hacer esfuerzos por rescatar la pedagogía de lo obvio, de aquellas acciones que hacemos y que por ser parte de la vida cotidiana ni siquiera las consideramos.

Las personas profesionales o investigadoras, tendremos que fomentar los pensamientos críticos y decodificar nuestras propias prácticas. Para ello, tenemos que comprender que la resolución de los problemas, ya no pasaría por la "receta" teórica, sino por el criterio de nuestra observación, curiosidad y asombro, principios que parecen obvios, pero que suelen perderse en el camino.

\footnotetext{
${ }^{5}$ Moreno (2002) ya lo detalla cuando explica como los padres piensan en otros entes socializadores como los medios de comunicación, el turista en la zona de Guanacaste y las oportunidades económicas (desempleo), el consumismo, la violencia familiar.

${ }^{6}$ Cultura es un conjunto de prácticas, de técnicas, de símbolos y de valores que se transmiten a las nuevas generaciones y garantizan la reproducción de un estado de coexistencia social. Se parte de una integralidad de factores sociales y personales donde se conjugan con prácticas heredadas.
} 
Por supuesto que dicho cambio implica construir las oportunidades para potenciar creativamente respuestas más acordes a las necesidades de amplios sectores de la población. Esta es una tarea que demanda también la compañía y la consulta con otras personas, sean profesionales o no, con el objetivo de defender y entender los desafíos a que nos enfrentamos.

Desde una posición socio-histórica, Vygotsky estudiado por Wertsch, (1995), nos indicaba que los procesos psicológicos superiores se desarrollan a partir de los procesos sociales. Los procesos mentales o psicológicos, integran los instrumentos y los signos culturales como mediadores del aprendizaje; por ello, en este trabajo se argumenta que a la vida humana no es posible homogenizarla.

Por ejemplo, este autor crítica la idea de la edad mental como una variable común. Comprueba que las diferencias en los procesos de desarrollo, en niños que fueron medidos a partir de pruebas psicométricas con "igualdad" en su edad mental, son distintas. E incluso critica el concepto de Piaget sobre los procesos intrapsíquicos o cognoscitivos.

En lugar de suponer que un único conjunto de principios explicativos, como la adaptación y la equilibración, pueden dar cuenta de todos los aspectos del desarrollo cognitivo, Vygotsky defendía que estos principios debían incorporarse en un marco explicativo más amplio capaz también de tratar los fenómenos socioculturales. (Wertsch, 1995, pp. 59)

Entonces, para Vygotsky no es tan importante dar cuenta de las fases del desarrollo como individuo aislado, sino que lo importante es estudiar cómo se gestan las relaciones cambiantes, entre las fuerzas del desarrollo y el conjunto de las posibles situaciones en que se llevan a cabo. Esta postura teórica tiene sus implicaciones metodológicas que afectan directamente el acercamiento a los acontecimientos humanos. Así, es importante observar al sujeto como tal, pero a la vez, es determinante comprenderlo en su interacción y evolución en el mundo sociocultural y a partir de las condiciones concretas en que se desarrolla.

Las prácticas profesionales, al menos en psicología y pedagogía, influidas por las tendencias a encontrar productos y no entender los procesos, nos han creado una representación abstracta de los procesos de crianza y socialización humana. Dicha postura ha invadido ámbitos tan importantes como las políticas y acciones gubernamentales, que instituyen 
normas, las cuales regulan la formación humana y no siempre son oportunas ni responden a la dinámica social.

Ante ello se requiere romper con las pretensiones de la universalidad, del etnocentrismo y del adultocentrismo, con el fin de reconocer la complejidad y diversidad, así como para develar las formas de dominio y poder que se instauran en los programas sociales. Entender cuál es nuestra representación de lo que hacemos en cuanto a las conceptuaciones teóricas y metodológicas, así como las posturas éticas ante la vida, nos debería llevar a observar críticamente nuestra propia práctica profesional y académica.

Rogoff (1993), por su parte, criticaba los estudios sobre desarrollo cognitivo en la niñez, los cuales, han girado alrededor de la tarea, aproximándose más a los aspectos específicos de la actividad cognitiva. Los límites de dichos estudios se relacionan con la falta de consideración del contexto en el cual el individuo está vinculándose con las otras personas y, de las instituciones sociales que normalizan la vida social en el marco cultural que en se dan las acciones. Por tal razón, menciona esta autora, que se tiene que establecer una perspectiva mucho más amplia, que ubique las características de la tarea y del acto cognitivo en función de la meta de la actividad y del contexto interpersonal y sociocultural en el cual se desarrolla.

En las prácticas, hemos optado por una conceptuación simplista e individualista de los sujetos humanos, ya Sampson (1996) lo ha planteado, cuando nos explica, desde la psicología, que se ofrece una visión de ser humano en abstracto y autocontenido en sí mismo, lo que nos hace caer en un monologismo o, podríamos decir, en un espejismo.

He dado el término de monologismo a este camino escogido para enfatizar el grado en que una minoría histórica de la población entera del mundo ha perseguido reducir las diferencias a una homogeneidad inquietante... y sin darle nunca al otro una oportunidad genuina para ser escuchado. (Sampson, 1996, pp. 48).

Dicha perspectiva es conveniente para un sistema social excluyente, que por ejemplo, tiende a culpabilizar y colocar la resolución de los problemas sociales en el cambio individual y en el psicologismo que ya Martín-Baró (1992) nos lo explicaba. Esto implica desconocer la 
dinámica social, de la cual somos parte. Así la dicotomía entre lo individual versus lo social, nos hace perder de vista la integración de los procesos humanos.

Víquez (2005), por ejemplo, al estudiar la ruta crítica de la niñez hacia la institucionalización, en el Patronato Nacional de la Infancia, demuestra cómo prevalece la "doctrina de situación irregular", cuyas consecuencias más evidentes están en la fragmentación de los procesos institucionales; en el manejo burocrático de los casos; en la depositación de la culpa en la figura materna; en la visión causal de las situaciones de vulnerabilidad social producto de las condiciones de pobreza y abuso y en las condiciones individuales, relacionadas con la edad y la limitación en cuanto a las opciones.

La doctrina de situación irregular, considera las problemáticas que aquejan a la niñez y sus familias, producto de una situación de "disfunción" familiar y por supuesto no se identifican las condiciones en que viven las personas, como una violación de derechos humanos. Por tal razón, se etiqueta y patologiza a los grupos sociales, los cuales, vienen a considerarse como problemáticos para la sociedad.

De allí que el sistema social, ubica la causa en los individuos o grupos, los cuales son juzgados por sus actuaciones y doblemente victimizados, en especial si provienen de condiciones adversas que los vulnerabilizan (Claramunt 2005a y b).

Por el contrario, la doctrina de protección integral, identifica a los derechos humanos como irrenunciables, universales e integrales, donde ningún derecho está sobre otro y todos tienen que ser garantizados. Esta perspectiva que está mucho más desarrollada en Claramunt (2005a y b) reta la práctica institucional, especialmente a partir de los años noventa, con la ratificación, por parte de Costa Rica, de la Convención sobre los Derechos del Niño (1990), la creación del Código de la Niñez y la adolescencia en el año 1998, entre otros.

En la actualidad, se viene ejecutando una reforma institucional en el PANI cuya tarea, se encuentra en el cambio de su enfoque y por consiguiente en las prácticas que se han venido realizando, tarea nada fácil, pues la costumbre y lo obvio ha sido la institucionalización y la visión de los problemas de niñez, revictimizando y agudizando en algunos casos el problema, más que resolviéndolo. 
Desde el enfoque que abogamos, tenemos la tarea inminente de revisar los procesos macrosociales, tales como la distribución de la riqueza, la pobreza, las oportunidades de educación, los servicios de salud, el empleo y el desempleo, lo multicultural y el tipo de Estado, para comprender mejor la vida de la niñez, de las mujeres, de las familias y de las comunidades, así como exigir el cumplimiento de los acuerdos internacionales.

Esto nos lleva a preguntarnos ¿cuáles son las oportunidades, que, como sociedad estamos construyendo para lograr el bienestar y el desarrollo óptimo? Y si estará efectivamente impulsando lo que indica, al menos, la definición del desarrollo humano sostenible, expuesta por el PNUD (2003) y su congruencia con el enfoque de protección integral. O por el contrario, si estamos tan influidos por una práctica instrumental y técnica que pierde de vista las particularidades, encasillando la realidad bajo un monologismo que nos hace ciegos(as), sordos(as) y mudos(as).

"El desarrollo humano es un proceso mediante el cual se amplían las oportunidades de los individuos; entre estas, las más importantes son una vida prolongada y saludable, acceso a la educación y el disfrute de un nivel de vida decente" (pp. 34, recuadro 1.4)

Entre otros muchos temas, cabe cuestionar la tendencia actual de instrumentalizar a la niñez y de seudo institucionalizarla a través del ingreso temprano al sistema educativo formal. Se ha de recordar el problema que surge por el acuerdo de la Sala Constitucional costarricense, cuando obliga al Ministerio de Educación Pública, a realizar un prueba para medir sí, un niño o una niña, de menor edad, está lista para ingresar al sistema educativo formal; respondiendo a la tendencia social de introducir rápidamente a la niñez a las aulas escolares, olvidando que no solo allí se educa la juventud (La Nación, sección El País, 1610-05). En este caso, queda pendiente, un estudio longitudinal que nos presente las consecuencias para la niñez, de este tipo de acciones que podría, entorpecer o no, el óptimo desarrollo infantil.

\section{Programas y programas.}

Desde la situación descrita, nos encontramos que las prácticas institucionales están siendo atomistas y simplistas, las y los profesionales de las ciencias humanas caemos en un ejercicio inerte de acciones, que responden por un lado a las demandas de un sistema 
institucional plagado de caminos burocráticos, que obstaculizan la creación de respuestas oportunas y atinadas a las necesidades sociales.

Somos las y los técnicos sociales, que brindamos sugerencias y definimos programas para la atención infantil; asumimos una posición de poder que mantiene la idea, muchas veces implícita, de que las personas al no tener formación educativa, o por ser pobres, o al estar necesitados, están incapacitadas para actuar o dirigir sus vidas y, por ello, se necesita de nuestras instrucciones. Esto elimina la posibilidad de realizar un diálogo ${ }^{7}$ con las personas directamente involucradas, perdiendo la oportunidad de construir conjuntamente los conocimientos.

El sistema social capitalista, en nombre de la globalización ${ }^{8}$, viene estableciendo políticas públicas de corte similar en todos los países del área, cuyos programas se implementan a partir de "modelos" estandarizados, bajo el principio de la replicabilidad, sin entender las maravillosas particularidades de los seres humanos. Cordero, (2004) lo ha planteado con respecto a una serie de ideas sobre las políticas de educación inicial, ejemplificado en el caso de las Madres-maestras ${ }^{9}$ en Panamá y la pugna con el gobierno de dicho país.

Continuando, con la controversia, vamos a presentar una revisión bibliográfica, que también da cuenta de las dificultades de implementar un programa de educación preescolar comunitario; y para ello, se utiliza como fuente inicial el reporte del programa del Colombiano, el cual inspira, el "modelo" a los Hogares Comunitarios en Costa Rica.

\subsection{Programas educativos no formales.}

Para Córmack y Fujimoto-Gómez (1993) y Fujimoto-Gómez (2000) en América Latina desde los años setenta, se han desarrollado, experiencias de educación preescolar no formales en al menos el $78 \%$ de los países de la región.

\footnotetext{
7 "En un diálogo genuino, dos personas con posiciones distintas se encuentran, cada una con su punto de vista intacto, y entran en un proceso compartido igualitariamente de definición, descubrimiento y ajuste" (Sampson, 1996, p. 42)

${ }^{8}$ La globalización se convierte en una argumentación para implantar los "modelos", en todas las latitudes del mundo, como si fueran la opción para resolver problemáticas.

${ }^{9}$ Programa de educación inicial no convencional, liderada y protagonizada, por grupos de mujeres en conjunto con las familias beneficiarias del servicio que de manera organizada y colectiva, realizan actividades de educación preescolar, en Cordero (2005) se puede encontrar mayor información.
} 
Ambas autoras, consideran como logros los siguientes aspectos: la atención e incorporación de niños y de niñas de las zonas pobres; integración de amplios sectores y comunidades que se reúnen para conseguir mejores condiciones de vida para la niñez; la creación de tecnologías apropiadas para la participación de los agentes educativos y la participación activa de los padres como los educadores principales de sus hijos e hijas.

Agregan que se han convertido en programas "democráticos, integrales, integradores, autogestionarios y participativos, los que se configuran como una alternativa válida para las zonas urbano marginales y rurales"(Córmack y Fujimoto-Gómez, 1993, p 104). Y otro aspecto a su favor, es que son opciones de menor costo, razón por la cual muchos gobiernos han intentado impulsarlos.

En cuanto a las limitaciones señalan: problemas en la capacitación; el asunto del no pago de los agentes educativos y distorsiones en las concepciones filosóficas. Además, comentan, que las experiencias han logrado realizar sus acciones por el financiamiento externo que han recibido y por la reunión de esfuerzos conjuntos de parte de los participantes, pero que ello es un problema su posible sostenibilidad.

Sugieren Córmack y Fujimoto-Gómez (1993) que para llevar a cabo programas de este tipo, han de tomarse en cuenta las siguientes recomendaciones, que nos parecen, que se dirigen a las instituciones gubernamentales, más que a los grupos comunales. Los programas, forman grupos de estudio sobre las problemáticas de la comunidad en general y de la niñez en particular. Proceso que requiere de una estrategia de trabajo comunitario. Así la presencia del agente externo está en su rol dinamizador, respetando los ritmos y condiciones de cada comunidad; con lo que se buscaría instrumentar a los grupos locales y a las comunidades para que hagan diagnósticos de la realidad y que ello les permita formular proyectos.

Asimismo, se sugiere hacer procesos de sensibilización de la población, utilizando fuentes diversas, como los medios masivos de comunicación.

La participación de los padres de familia es necesaria, pues la programación y ejecución recae sobre ellos, desde una perspectiva abierta. En algunos proyectos los agentes educativos son propuestos directamente por las comunidades. 
La capacitación tiene que ser constantes y permanente para garantizar la calidad del servicio, en el campo pedagógico, de salud y administración. Se sugiere tener "paquetes" de instrucción y de evaluación, que posteriormente sirven para evaluar la experiencia. Por ello es necesario que exista una articulación de acciones en las distintas modalidades educativas.

De manera particular, se recomienda la designación de instituciones que realicen la sistematización de las experiencias, así como la incorporación y la presencia de estudiantes universitarios, bajo la modalidad de servicio social, para que puedan aportar a los procesos.

Promover la coordinación interinstitucional para enfrentar problemáticas familiares en campos tan diversos, como los servicios de salud, educación, producción agrícola, trabajo, vivienda y otros. Centralizar la información para ser consultada por las instituciones que así lo requieran.

Y unido a todo lo anterior, se sugiere la búsqueda de recursos de la cooperación internacional y promover recursos internos.

De las sugerencias se desprende la necesidad de un ente coordinador eficaz y comprometido, que articule e integre los recursos tanto internos como externos y que permita el seguimiento y continuidad, respetando los procesos locales.

De igual manera, agregamos que el trabajo con las comunidades implican procesos diversos y complejos; por esta razón, es muy importante ubicar las experiencias dentro de un campo de acción participativo, que conceptúan el trabajo con los adultos, niños, niñas y organizaciones, así como la definición de acciones en el campo de la investigación.

En este sentido, en América Latina ya se ha planteado una agenda investigativa relacionada con la formación de los y las educadores; el financiamiento de parte de los gobiernos ${ }^{10}$; la coordinación con las organizaciones de la sociedad civil ${ }^{11}$; la implementación de programas

\footnotetext{
10 "Sigue existiendo una fuerte sensación de que este ámbito está mal financiado, de que la ayuda financiera pública es muy baja e inestable, y de que la carencia de recursos es un problema importante" Myers (2000) p 29.

${ }^{11}$ No hay coordinaciones entre gobiernos y experiencias locales. 
en poblaciones de escasos recursos ${ }^{12}$ y en las zonas rurales; la creación de currículos integradores que tomen en cuenta las condiciones culturales ${ }^{13}$, geográficas, económicas y la edad; la des-burocratización y la calidad de los programas; el seguimiento y las evaluaciones pertinentes a corto y largo plazo. Grandes áreas de investigación que corresponden, desde mi punto de vista, en deficiencias encontradas en el campo y que requieren de esfuerzos más sistemáticos y coordinados, los cuales siguen siendo urgentes.

\subsection{La experiencia colombiana que inspira a los Hogares Comunitarios en Costa Rica.}

Amar (1994) nos señala que, el enfoque de trabajo de los programas realizados en Colombia se vinculan directamente con el desarrollo comunitario y aglutinan la participación de varias organizaciones ${ }^{14}$ gubernamentales, civiles, comunitarias, privadas y familiares que toman como eje la promoción y la atención infantil. Entre sus principios y condiciones identifica los siguientes:

1. El centro de atención se da a partir del niño o la niña en su contexto y mundo de relación social. De forma paralela se tiene como eje fundamental el desarrollo comunitario; se parte del supuesto de que es imposible apoyar a la niñez pobre, si no se trabaja integralmente, en las comunidades, atendiendo procesos simultáneos. Tal como se conceptúa seguidamente:

Se concibe la atención integral al niño como un proceso social que contribuye efectivamente al desarrollo integral de éste. Esto implica una serie de servicios básicos como la nutrición, educación, salud, recreación y concientización de la comunidad como gestora de cambio de las condiciones de vida (Amar, 1994 p 317)

\footnotetext{
${ }^{12} \mathrm{~A}$ pesar de los esfuerzos por dirigir acciones hacia los grupos con ingresos bajos, "es probable que exista un fuerte sesgo en los programas en contra de la población con ingresos bajos en la mayoría de los países" Myers (2000), p 24.

${ }^{13}$ Se puede revisar el trabajo de Cordero (2003) que plantea algunos desencuentros entre las prácticas escolares y las familiares.

${ }^{14}$ Entre ellas se menciona el Instituto Colombiano de Bienestar Familiar (ICBF), la Universidad del Norte de Barranquilla, la Fundación Bernard van Leer, soportado por programas de grado en Educación infantil, psicología, enfermería y las maestrías en desarrollo social y familiar 
2. Los proyectos, se realizaron por la reunión de acciones conjuntas, entre los pobladores de las comunidades y otros entes. La Universidad del Norte de Barranquilla, tuvo un papel fundamental al organizar y promocionar el desarrollo comunitario alrededor de varios recursos institucionales y comunales. A la base de la experiencia los pobladores, contaron con programas de investigación y la participación de estudiantes de las carreras de psicología, educación y enfermería, que ofrecieron el recurso humano para apoyar las acciones de organización, entre otros.

3. Otro de los ejes fue la participación de las familias como agentes educativos directos, entendidos por su importancia como vínculos primarios en la vida humana.

4. Desde la Universidad, se movilizaron recursos y se estableció como básico la participación democrática de las comunidades, con el fin que la presencia de los pobladores, fuera más allá de la atención infantil, propiciando acciones positivas para el desarrollo comunitario. Se consiguieron recursos gubernamentales, y se logró el reconocimiento del programa.

En cuanto a los principios se enuncian los siguientes:

a) Una educación que parte de las necesidades de los pobladores, por esta razón, el diagnóstico participativo se constituyó en un espacio donde los lugareños y los profesionales compartían.

b) Se conceptuó la educación a partir de la participación de todos los involucrados, tomando como supuesto, que en toda acción humana se desarrollan aprendizajes y que todos podemos contribuir en la educación infantil.

c) La conciencia de la educación permanente, potenció otros espacios donde se incorporaron los habitantes a programas de educación formal y no formal.

d) Se buscó la calidad educativa, la cual se entendió como la integración de la realidad y los aspectos aportados por la universidad a partir de procesos metodológicos. En este sentido, es interesante que Amar (1994) presenta una serie de críticas a la psicología del desarrollo infantil, sin embargo, para sustentar algunos de los supuestos de trabajo, se observa una visión patológica de lo que acontece en las comunidades pobres y con la niñez.

e) La meta de una educación integral para el preescolar, se basó en la identificación de las necesidades básicas en diferentes dimensiones personales y sociales (biológicas, psíquicas, educativas y económicas sociales). 
f) La vinculación universitaria más cercana a los problemas comunales, contribuyó a la docencia, la investigación y la acción social.

Siguiendo los principios mencionados y considerando el desarrollo comunal como uno de sus fines, se impulsó el trabajo con la niñez como un aspecto fundamental de la comunidad; así como la participación de las familias y la conciencia de su papel; la organización de los centros a cargo de una mujer respetada y escogida por la comunidad, con el apoyo de una joven y la presencia de una madre voluntaria por día, con el objetivo de atender las necesidades educativas y de nutrición de los niños menores de siete años, en grupos de 25 niños y niñas. De acuerdo, a lo reportado por Amar (1994) se habían logrado contar con 127 Hogares Comunales, los cuales funcionaban en las casas de habitación de las mujeres.

\subsection{Hogares Comunitarios en Costa Rica}

Tal como se mencionó, el siguiente apartado proviene de una revisión bibliográfica del programa y no por la participación directa de parte de la autora. En este sentido, es una mirada específica y tiene el objetivo de ofrecerle, al lector o lectora, una perspectiva, un ejemplo basado en documentos. Por tal razón, se motiva a otras personas a responder o escribir sobre el tema.

Tal cual hemos descrito el programa colombiano, damos por entendido que fue una fuente de inspiración importante para justificar el programa de Hogares Comunitarios, en Costa Rica. No obstante, la implementación no respondió al "modelo" sobre todo porque el ámbito del desarrollo comunitario fue el más débil.

En esta línea Brade (Cordero, 2006b) explica que los planteamientos políticos sobre el programa, en los años noventa, no necesariamente correspondieron al proceso institucional que lo ejecutó y a las limitaciones de la ejecución. Por ello se plantea la importancia de reflexionar sobre este tipo de experiencias y realizar cambios en las prácticas institucionales, con el objetivo mejorar los procesos institucionales que tienen como misión apoyar la organización comunitaria.

Desde nuestra interpretación otro factor que influyó el programa es el modelo centrado en el adulto (Chavajal y Rogoff, 1999 y 2002; Matusov y Rogoff, 1999; Rogoff, 1997; Rogoff y Toma, 1997: Rogoff, Matusov y Write, 1996; Cordero. 2005), cuya figura central es la 
persona encargada de la niñez, quien desde una posición de poder indica y define el proceso de trabajo, controlando todo el proceso. Así el programa contacta en las comunidades a una mujer que se encarga de los niños y las niñas, pero tal como se encuentra en los estudios realizados en la Universidad de Costa Rica, el programa no responde a los principios, entre ellos la búsqueda de una corresponsabilidad comunitaria.

Los Hogares Comunitarios en Costa Rica, surgen por iniciativa de la Primera Dama de la República Doña Gloria Bejarano de Calderón en el gobierno de Rafael Ángel Calderón 19901994, están a cargo del Instituto Mixto de Ayuda Social (IMAS). Las características de los programas, de acuerdo a la información revisada en la página web (http://www.oei.org.co/quipu/costrica/cost07.pdf revisada en el año 2001 y 2006 ${ }^{15}$ ) correspondiente a la posición oficial del Gobierno de Costa Rica, como es la siguiente:

1. En el programa participan la comunidad, el Ministerio de Educación, Ministerio de Salud, Ministerio de Trabajo y el IMAS.

2. Comunidad, Estado y Organizaciones internacionales ejecutan el programa.

3. La comunidad escoge a las Madres comunitarias, sin embargo, ellas para ser aceptadas son entrevistadas, se les hace una valoración psicológica y un estudio social.

4. Reciben un curso de cuarenta horas en temas de desarrollo infantil, nutrición, organización, actividades educativas, atención de emergencia y otros.

5. Para que el hogar funcione se les hace préstamos blandos para que arreglen sus casas.

6. La cantidad de niños y niñas es de diez, con un horario de 6 de la mañana a 6 de la tarde. El compromiso de la madre comunitaria es brindar cuidado, alimentación, educación y sobre todo cariño.

7. Los padres de familia usuarios del servicio, pagan un monto mínimo y aportan 2 frutas, verduras frescas y dan 4 horas voluntarias por semana, ellos o algún familiar en el hogar.

8. El Programa Mundial de Alimentos y el Fondo de Asignaciones Familiares brinda apoyo y algunos alimentos.

\footnotetext{
15 Para Brade (Cordero, 2006) esta información esta desactualizada, pues UNICEF desde hace muchos años no hace este tipo de donaciones.
} 
9. UNICEF entrega vajillas, sillas, mesas, utensilios de cocina, materiales didácticos y otros. Además apoya en capacitación e investigación, junto a organizaciones no gubernamentales.

10. Las organizaciones comunitarias supervisan, apoyan y canalizan los recursos materiales.

La Universidad de Costa Rica, a partir de dos tesis realizadas en la Escuela de Enfermería interesadas en la nutrición infantil; tres en el ámbito educativo y una en la Escuela de Sociología y Antropología, realiza un estudio del programa evidenciando la pérdida de rumbo.

Arce; Arce; Brenes; Camacho; Meléndez y Mora, (1994) al comparar el programa con otros, concluyen que si bien las diferencias en la evaluación efectuada sobre el desarrollo a 318 niños y niñas no muestran diferencias significativas, sí hay serias deficiencias administrativas y metodológicas. No se poseen programas educativos con objetivos y estrategias, para que el docente o encargada desarrolle actividades sistemáticas y específicas a cada nivel.

Asimismo, en los Hogares Comunitarios hay un inadecuado aprovechamiento de los recursos humanos y materiales, por no contar las madres comunitarias con una supervisión ni preparación constante. El espacio físico no es adecuado para que el infante pueda accionar libremente. Además, enfatizan en el riesgo que existe en la integridad física y psicológica de los niños y niñas por la presencia de personas ajenas al programa. De igual manera, ubican como negativo la participación de niños de edad escolar en el espacio del hogar.

Rodríguez (1999), cuando hace un análisis sobre la forma de supervisión que se realiza en el programa, concluye que en la mayoría de los casos la función está directamente relacionada con la fiscalización, inspección y cumplimiento de normas establecidas. En este sentido, las supervisoras utilizan una fórmula de control de planilla para justificar el subsidio alimentario que recibe cada hogar. La mayor debilidad de la supervisión es la ausencia de capacitación específica a las supervisoras y a las entidades de apoyo.

A parte de ello, explican que no hay un apoyo efectivo de parte de organizaciones comunales, las cuales en un $66 \%$ desconocen el programa. Se demuestra que en la práctica 
el fin organizativo que inspiró la propuesta colombiana no se cumple. Esta misma conclusión la mantiene Benavides y Soto (1999), que a su vez agrega que también el IMAS presenta un apoyo limitado, con una falta de seguimiento al programa y con un papel represivo, ya que no se recibe el subsidio, por la inasistencia de un niño o una niña.

Benavides y Soto (1999), estudian las familias que participan del servicio; concluyen que no hay una selección técnica para escoger a las familias, recayendo en la Madre comunitaria la tarea, la cual es cumplida a partir de sus criterios personales. Reconocen el hecho que los niños y niñas participantes provienen de hogares donde la madre es la jefa de hogar. Esta situación, explica el hecho que sean mujeres con una doble jornada laboral y que su única opción está en enviar a sus hijos(as) a los Hogares Comunitarios. De la misma manera, son féminas violentadas en su historia biográfica y, por tal razón, no confían en sus familiares para cuidar de los menores. En el Hogar Comunitario se logran resolver las necesidades primarias o básicas, de alimentación, cuidado, afecto e higiene, no así, necesidades más complejas como el desarrollo cognitivo.

La capacitación a las Madres comunitarias es una necesidad no resuelta, por otra parte, no hay una dotación básica de materiales, ni existe una atención diferencia según la edad, hay muchos menores en un espacio físico reducido. Se suele responsabilizar a los niños y niñas mayores de los más pequeños y los programas de televisión que ven, no siempre son del agrado de los padres de familia. Además perciben desmotivación y cansancio en la Madre comunitaria y se destaca que en el hogar hay otras personas no vinculadas al programa, coincidiendo con Arce, et al (1994).

Los trabajos de Ortiz (1997) al capacitar a un grupo de Madres comunitarias, y el de Araya, Espinoza y Picado (1999) al incorporar a niños con discapacidades, por medio de asesoramiento directo a la madre biológica y comunitaria, demuestran cómo existe un interés genuino de parte de las encargadas de los hogares por involucrarse en apoyos de este tipo. Evidentemente, la tarea de cuidar niños y niños requiere de una serie de recursos pedagógicos, que tendrían que estar desarrollados constante y permanentemente, para no perjudicar la labor.

Gutiérrez, Marín y Spence (1999) si bien les interesaba evaluar la salud de los infantes en quince hogares, concluyen que las encargadas no organizan las actividades de acuerdo a 
los requerimientos del programa, el interés se concentra en la alimentación, recreación, estimulación y descanso. En el tema nutrición aún existe una población de mayor riesgo y, las niñas presentan una tendencia a la desnutrición y los varones al sobrepeso.

La cuarta parte de los infantes no tiene una cobertura de seguridad social y el $12 \%$ de ellos tiene un esquema de vacunación incompleto, siendo en su mayoría, población emigrante nicaragüense. Situación que atenta contra los derechos de la niñez y que irrespeta el código de la niñez y la adolescencia costarricense. No hay un compromiso real de cooperación continua de parte de las instituciones estatales, siendo una situación que atenta contra las garantías sociales para la población infantil. No obstante, en opinión de las autoras, es una solución positiva de atención a la niñez en riesgo social, pues al menos, se le brinda a los usuarios cariño, apego, valores morales y espirituales.

Sin querer caer en una visión sobre el programa de Hogares Comunitarios desde las encargadas directas y las comunidades, lo importante que me interesa destacar, más bien, es la participación de las instituciones gubernamentales, que lejos de convertirse en un ente coordinador y facilitador de procesos, se constituyen en un accionar burocrático que pierde el rumbo. Esto nos lleva a cuestionar la dinámica institucional que no logra realizar un efectivo trabajo y que evidentemente tiene la tarea de cambiar su dinámica.

\section{Señalamientos y alternativas.}

Para construir alternativas y procesos integradores, en las prácticas de investigación y en el campo de acción profesional, requerimos al menos considerar los siguientes aspectos, los cuales se puntualizan sin ser "recetas" ya que se tiene que tomar en cuenta las particularidades, condiciones y diversidad de los procesos humanos.

1. Nunca perder de vista la perspectiva de protección integral de derechos y, por ello, hay que hacer un esfuerzo por cambiar el enfoque de situación irregular, que suele identificar a las poblaciones de manera patológica por sus actuaciones.

2. Reconocer la doble dimensión de los sujetos humanos en su concreción individual así como representantes de la vida en sociedad. Esto implica no solo considerar a la persona como tal, sino entender el marco en donde se desarrollan los procesos humanos, por ejemplo la socialización infantil, tomando en cuenta las condiciones en que es posible la crianza y, el marco socio-cultural que le da sentido a las acciones. 
3. Concebir las prácticas sociales como procesos, más que como productos acabados. Se parte de la complejidad de la realidad social y se considera el movimiento y la transformación como parte cosustancial a los procesos humanos, como nos lo indica Jara (2003, p. 1) "Las experiencias siempre están en movimiento y en toda experiencia intervienen muchos elementos: condiciones, situaciones, acciones, percepciones, emociones...".

4. Entender que las personas nos involucramos en los procesos sociales (de los cuales formamos parte) y considerar la pretendida neutralidad u objetividad instrumental, como una pretensión imposible de cumplir. Siempre que participamos en acciones humanas, estamos sintiendo emociones y realizamos interpretaciones valorativas, que parten de nuestro marco de referencia. Por eso tenemos que tener una mirada crítica a nuestras propias actuaciones y buscar la discusión y la reflexión con otras personas que puedan ofrecer otras posiciones y enriquecer los procesos a partir de la diversidad.

5. Concebir la formación humana siempre, en relación con otros y con la naturaleza, en una complejidad, cuyo impacto directo se realiza en la conformación de la identidad social e individual (Martín-Baró, 1992).

6. Tal como lo ha planteado Ibáñez (1994), tenemos que comprender que lo colectivo (grupal) no se puede homologar con lo social, ya que las particularidades culturales, los modos de vida aprendidos, las metas y las normas acordadas, así como, las condiciones materiales están de manera dinámica en la vida social.

7. En el campo del desarrollo infantil, las relaciones madre-hijo(a) son importantes, pero éstas no pueden ser entendidas como las únicas relaciones. Se ha de entender que la vida social incluye al marco de las condiciones en que se desenvuelve la niñez, la vinculación con todas personas cercanas y las oportunidades a que se tiene acceso. En este sentido, hemos de comprender la importancia de otros agentes socializadores como son los medios de comunicación colectiva, el grupo de pares, el barrio y la escuela, entre otros (Moreno, 2002). Ana Teresa Álvarez (Cordero, 2006a) quien al conversar sobre la investigación realizada en los años ochenta de Álvarez, Brenes y Cabezas, (1991) coincide con la crítica que se hace en este trabajo y, comenta que en aquella ocasión, no se logró establecer una metodología acorde con el planteamiento teórico más amplio que se planteaba.

8. Entender que partimos de un mundo de ideas, creencias y conceptuaciones construidas a partir de las prácticas culturales las cuales, suelen estar incorporadas 
en la subjetividad humana a partir de teóricas implícitas y explícitas que influyen en la crianza infantil o en el trabajo comunitario y, entender que estamos influidos por nuestro imaginario social de como hemos aprendido que es la vida en sociedad.

9. Comprender que la vida humana no esta programada y que muchas prácticas cotidianas responden a las condiciones y vivencias de la experiencia que se realiza, en función del medio en que se desarrollan. Por tal razón, hemos de fomentar la capacidad de observación de aquellas situaciones y momentos, de la rutina cotidiana, así como las distintas formas en que se reproduce la vida como es en lo que se entiende por diversión, el ocio, trabajo y compañía, que nos hace ser en el mundo.

10. Por lo anterior, existe la obligación de considerar el contexto social más amplio en el que se organizan las actividades infantiles y nuestra práctica profesional. La memoria, el lenguaje, el manejo de instrumentos, la planificación están estrechamente ligadas a las prácticas culturales y no son motivadas sólo, por factores intrapsíquicos.

11. Se tienen que estudiar las prácticas culturales de la crianza infantil y de nuestra propia práctica institucional tomando en cuenta las particulares en que se desarrollan. Así la forma en que se utiliza el lenguaje y bajo que fórmulas se realizan, no responde a un carácter universal, sino que se hacen dependiendo del contexto y del significado que el grupo de referencia establezca.

12. Los estudios teóricos sobre el desarrollo infantil son importantes, pero hemos de tener una perspectiva crítica para introducir las particularidades y entender los procesos que observamos. Más que pensar en etiquetas diagnósticas tendríamos que lograr comprender los procesos de desarrollo, para abrir oportunidades, antes que cerrarlas. Entender las lógicas de trabajo que hemos venido realizando y evaluar nuestras actuaciones para realizar cambios positivos.

13. Una tarea permanente es la ruptura con visiones rígidas y estandarizadas de cómo es el desarrollo infantil. Esto implica relativizar de conceptuaciones que estandarizadas al ser humano y de aquellas que nos presentan un individuo en abstracto, un niño o niña de "libro". .

14. Se tiene que entender que la participación y los cambios son posibles cuando se comparte con las y los involucrados directos, cuando se dialoga, cuando como sociedad nos comprometemos en la crianza infantil $y$, cuando respetamos los derechos humanos, como principios integradores en el campo de la educación; de la vida en compañía (familia); de la salud; de la vivienda; del respeto a la identidad, entre otros. Esto significa construir procesos con las y los involucrados para crear 
acciones y gestionar posibilidades, integrando los aportes de todas las personas en equidad de condiciones.

15. Ello nos demanda identificar las formas de organización social que estructuran las actividades infantiles, todo ello afectado, como dice Rogoff (1993), por la elección del rol y por la estructura social en que se marcan las posibilidades de acción de las prácticas culturales.

\section{Conclusiones}

Con grandes esfuerzos y sinceras buenas intenciones las instituciones sociales y académicas desarrollamos acciones para mejorar las condiciones de vida de la infancia en nuestro país. No obstante, algunas de estas visiones tienen el sesgo de identificar a la niñez desde la individualidad, excluyendo la histórica social y biográfica, así como obviando el contexto $^{16}$ socio-cultural. Por ello existe el desafío de construir prácticas investigativas y actividades gubernamentales articuladas, que tomen en cuenta a los sujetos involucrados y apunten a la integración de los procesos sociales, considerando la diversidad de las identidades.

Celebrar las diferencias requiere entender los procesos, más que llegar a productos cuantificables, es comprender que estamos en un marco de acción social amplio, constituido por redes sociales que le dan sentido a la forma de ser la especie humana. Tendencia social que esta reforzada por la mirada tecnificada de la vida humana. Por ejemplo, nuestros gobiernos a nivel político responden con números (en su más amplio sentido relacionados con estadísticas, costos y dinero) e intentan legitimar sus acciones a partir de ellos.

Por tal razón, hay un tendencia a controlar la complejidad de los fenómenos sociales y los procesos humanos; control que implícitamente plantea la homogenización de procesos, la replicación de "modelos" en contextos diferentes, bajo la idea que existe una cultura universal, invisibilizando las diferencias y justificando las discriminaciones.

\footnotetext{
${ }^{16}$ Usualmente el contexto se identifica por medio de variables externas y no se integra de manera comprensiva en la vida de las y los infantes. En el siguiente vínculo, se presenta la fotografía de un niño con su madre que esta separada en tres momentos. Usualmente, se da la tendencia a abstraer al infante (cuadro que despliega la presentación solo al infante), y, en el mejor de los casos estudiar la relación con la madre (niño y madre), excluyendo el ambiente donde se da la acción (niño, madre y vivienda) y a otros personas que suelen ser importantes en la vida infantil. No se toma en cuenta el contexto ni las condiciones. Esta explicación, ha sido retomada de las presentaciones que hace la Doctora Bárbara Rogoff (Cordero, 2002) en sus clases en la Universidad de California en Santa Cruz. 
Así es necesario romper con la "ilusión" que nos ha llevado a incorporar en las prácticas profesionales, la idea de la responsabilidad casi exclusiva de quien dirige o tiene a cargo un proyecto, sin considerar a los sujetos involucrados. La participación y la corresponsabilidad de las acciones nos indican entender a las y los beneficiados de los programas sociales, como sujetos que participan en los procesos, cuyas actuaciones no son ingenuas.

Es necesario realizar un esfuerzo colectivo por entender la responsabilidad social que tenemos en la constitución de una sociedad que cumpla con los ideales que se han propuesto en los Derechos Humanos. Por ello, hemos de revisar desde una posición crítica y comprometida las tendencias que nos vienen impulsando los procesos de globalización y los intereses de una sociedad capitalista. Se necesita tener más tino en la implementación de programas y debemos romper con la tecnificación de la socialización primaria. Por tal razón, hemos de problematizar la educación formalizada, por no decir escolarizada e institucionalizada, que pretende impulsarse desde el nacimiento una cobertura de enseñanza desde el preescolar (Ministerio de Educación) como si la formación humana se diera solo en las aulas escolares.

Hemos de tener una mirada crítica ante las Ciencias humanas $y$, en particular a la Psicología, que ha desarrollado una serie de interpretaciones y reinterpretaciones de infinidad de eventos, entre los cuales encontramos amplios tratados sobre la constitución del sujeto, sobre la construcción de la subjetividad y, por tanto, sobre la conformación de la sociedad. Pero dichas propuestas teóricas, aunque ofrezcan argumentos convincentes para las comunidades de científicos y profesionales, no siempre responden a las necesidades de sectores de la población.

Ante esto, se apela a la búsqueda de opciones alternativas, así como, propuestas creativas que nos ayuden a enfrentar situaciones complejas desde las prácticas investigativas y profesionales. Que tengan como objetivo la integración de concepciones distintas, afectivas y que permiten entender que nuestra tarea está, en facilitar y promover la construcción de redes sociales para el cuido y atención infantil. 


\section{Referencias}

Álvarez, Ana T.; Brenes, Abelardo \& Cabezas, Marylin. (1991). Patrones de crianza en la familia costarricense. San José, Universidad de Costa Rica, IIP: Actualidades en Psicología, 6(61): 1-590.

Amar Amar, José Juan. (1994). Educación infantil y desarrollo Social. Bogota: Ediciones UNINORTE.

Araya Abarca, Eileen; Espinoza Valverde, Ana Lorena y Picado Zeledón, Maritza. (1999). Capacitación a madres comunitarias para la participación de niños y niñas con retos múltiples en hogares comunitarios. Tesis para optar al grado de licenciatura en Orientación, Escuela de Orientación y Educación Especial, Facultad de Educación, Universidad de Costa Rica.

Arce Martínez, Elena; Arce Martínez, Floribeth; Brenes Rodríguez, Milagro; Camacho Zamora, Emma; Meléndez Delgado, Seidy y Mora Rodríguez, Nidia. (1994). Análisis comparativo de los Hogares Comunitarios con otras instituciones de Educación Preescolar. Tesis para optar al grado de licenciatura en preescolar: Escuela de Formación Docente. Facultad de Educación, Universidad de Costa Rica.

Benavides Rivas, Betzaida; Soto Zúñiga, Paul. (1999). Evaluación de los efectos sociales en la población beneficiaria del Programa Hogares Comunitarios IMAS en el área Metropolitana de San José. Tesis para optar al grado de licenciatura en antropología. Práctica dirigida en el IMAS, Escuelas de Sociología y Antropología. Facultad de Ciencias Sociales. Universidad de Costa Rica.

Carmiol Barboza, Ana María. (2003). La psicología cultural de la crianza: un estudio sobre las concepciones de las madres. Tesis por el grado de Licenciatura en Psicología, Escuela de Psicología, Facultad de Ciencias Sociales, Universidad de Costa Rica.

Chavajay, Pablo y Rogoff, Bárbara. (1999). Cultural variation in management of attention by children and their caregives. Developmental Psychological. 35(4): 1079-1090.

Chavajay, Pablo y Rogoff, Barbara. (2002). Schooling and traditional collaborative social organization of problem solving by Mayan mothers and children. Developmental Psychological. 38(1): 55-66.

Claramunt, Cecilia. (2005a). Explotación Sexual Comercial: Guía de trabajo para proveedores/as y encargados/as de servicios dirigidos a personas menores de edad víctimas. San José, Costa Rica: OIT/IPEC.

Claramunt, Cecilia. (2005b). Explotación Sexual Comercial y Trabajo Infantil. Análisis de la respuesta estatal costarricense a las personas menores de edad y sus familias desde el enfoque de los derechos humanos. Tesis Maestría en Psicología.- San José, C. R: Universidad de Costa Rica, Sistema de Estudios de Posgrado.

Consejo Episcopal Latinoamericano (CELAM) y UNICEF. (1994). Estudios de Prácticas de crianza: contexto general. Bogota: UNICEF, SELAC, CELAM. 
Cordero, Teresita. (2006a, marzo). Comunicación personal. Conversación informal con la investigadora Ana Teresa Álvarez, Instituto de Investigaciones Psicológicas, Universidad de Costa Rica.

Cordero, Teresita. (2006b, marzo). Comunicación personal. Conversación informal con la investigadora Krissia Brade, UNICEF-Costa Rica.

Cordero, Teresita. (2005). Experiencias pedagógicas de las Madres-maestras: comprendiendo los significados de las actividades del jardín. Tesis para optar al grado de doctorado en educación, Facultad de Educación, Universidad de Costa Rica.

Cordero, Teresita. (2004). Educación inicial en América Latina: situaciones y retos. Caso panameño. Revista Educación. 28(1): 39-53.

Cordero, Teresita. (2002). Comunicación personal. Conversaciones con la Tutora Rogoff, Barbara en Pasantía en la Universidad de California en Santa Cruz (UCSC), último trimestre del año.

Córmack Lynch, Maribel y Fujimoto-Gómez, Gaby. (1993). Estado del arte de la atención del niño menor de seis años en América Latina y el Caribe. PREDE/ O.E.A., Programa regional de Desarrollo Educativo, (10), Documento de trabajo del proyecto multinacional de Educación Básica (PRODEBAS).

Fujimoto, Gaby (2000). Modalidades alternativas en Educación Inicial. Especialista principal de Educación, Unidad de Desarrollo Social y Educación / OEA. Retomado de página web del Banco Mundial en Internet, sobre el simposio realizado en Chile en marzo del año 2000 www.worldbank.org/children/nino/basico/refs.htm

Gergen, K. (1996). Realidades y relaciones. Aproximaciones a la construcción social. Madrid, España: Editorial Paidós.

Gutiérrez, Yamileth; Marín, William y Spence, María. (1999). Impacto de los Hogares Comunitarios en el cuidado de la salud de la niñez en las comunidades de Alajuela centro, Escazú y Moravia. Tesis Escuela de Enfermería con énfasis en Salud de la Mujer y perinatología, Universidad de Costa Rica. Costa Rica.

Ibáñez, Thomas. (1994a). La psicología social como dispositivo desconstruccionista. En Psicología social construccionista (pp. 217-243). México: Publicación de la Universidad de Guadalajara

Ibáñez, Thomas. (1994b). ¿Cómo se puede no ser construccionista hoy en día? En Psicología social construccionista (pp. 245-257). México: Publicación de la Universidad de Guadalajara

Ibáñez, Thomas. (1994c). Construccionismo y psicología. En Psicología social construccionista (pp. 259-279). México: Publicación de la Universidad de Guadalajara

Instituto Mixto de Ayuda Social, (IMAS). (2001). Programa Hogares Comunitarios. En http://www.oei.org.co/quipu/costrica/cost07.pdf 
Jara, Oscar. (2003). Sistematización de experiencias: algunas precisiones. Pátzcuaro, México: Curso latinoamericano CREFAL. CEAAL: www.alforja.or.cr/sistem

Martín-Baró, Ignacio. (1992). Acción e ideología, psicología social desde Centroamérica. San Salvador, El Salvador: UCA Editores.

Matusov, Eugene y Rogoff, Barbara. (1999). Newcomers and oldtimers: educational philosophies-in-action of parent volunteers. Anthropology and Education Quarterly, 33(4), 1-26.

Ministerio de Educación Pública - Costa Rica y Organización de Estados Americanos (OEA). (1994). Patrones de Crianza de los Niños costarricenses de 0 a 4 años de edad. Costa Rica: MEP, Departamento de Educación Preescolar \& Multinacional de Educación Básica, OEA.

Ministerio de Educación Pública - Costa Rica y Organización de Estados Americanos (OEA). (1992). Patrones de Crianza de los Niños preescolares costarricenses. Costa Rica: MEP, Departamento de Educación Preescolar \& Multinacional de Educación Básica, OEA.

Ministerio de Educación Pública - Costa Rica. (2004). Preescolar. Recuperado el 28 de julio, 2005 de http://www.mep.go.cr/docentes/AntecedentesPreescolar.html

Myers, Robert G. (2000). Atención y desarrollo de la primera infancia en Latinoamérica y el Caribe: una revisión de los diez últimos años y una mirada hacia el futuro. Revista Iberoamericana de Educación. (22): 17-60.

Moreno, Moreno, Wagner. (2002). Procesos de crianza de Padres y Madres Guanacastecos, y su incidencia en la formación de valores familiares. Tesis Magíster Scientiae en Psicología, Sistema de Estudios de Posgrado, Universidad de Costa Rica.

La Nación. (2005). Prueba para preescolar se iniciará el 12 de diciembre. San José, Costa Rica: Sección El País día 16-10-05.

Ortiz, Pedro. (1997). Programa de educación para las madres encargadas de los Hogares Comunitarios en atención integral de la niña y el niño menores de seis años de edad en la Aurora de Heredia. Tesis en Enfermería con énfasis en Salud de la Mujer y perinatología, Universidad de Costa Rica.

Programa de las Naciones Unidades para el Desarrollo (PNUD). (2003). Segundo Informe sobre Desarrollo Humano en Centroamérica y Panamá. Costa Rica: Proyecto Estado de la Región, Editorama S.A.

Rodríguez, Claudia. (1999). La supervisión ejercida por las entidades de apoyo en el programa Hogares Comunitarios de Heredia. Tesis de Maestría de Educación Administrativa, Facultad de Educación, Universidad de Costa Rica.

Rogoff, Barbara. (2002). The cultural nature of human development. Oxford University Press. 
Rogoff, Bárbara. (1993). Aprendices del pensamiento, el desarrollo cognitivo en el contexto social. España: Paidos.

Rogoff, Barbara; Matusov, Eugene y Cynthia White. (1996). Models of Teaching and Learning: Participation in a Community of Learners. Handbook of education and human development. Oxford, UK: Blackwell.

Rogoff, Barbara y Toma, Chikako. (1997). Shared Thinking: Community and Institutional Variations. Ablex Publishing Corporation. Discourse Processes: 23, 471-497.

Rosabal, Mariano. (2004). Alternativas teórico-metodológicas para el estudio de la crianza: estado del arte. Documento de trabajo inédito, presentado al programa de la Universidad de Costa Rica PRIDENA.

Sampson, Edward. (1996). Celebrando al Otro: una interpretación dialógica de la naturaleza humana. Cordero, T., Dobles, I. y Pérez, R. (Comp.). Dominación social y subjetividad. Contribuciones de la psicología social. San José, Costa Rica: Editorial de la UCR.

Valverde, Hellen Roxana. (2005, 24 de noviembre). Presentación de libros: Secretos de la Educación de las Madres-maestras y Padres-maestros. San José, Costa Rica: Auditorio de Letras.

Wertsch, James. (1995). Vygotsky y la formación social de la mente. España: Editorial Paidós.

Víquez, Nioe (2005). Estado, familia y persona menor de edad. Análisis de la ruta crítica hacia la institucionalización de niños, niñas y adolescentes en Costa Rica. Tesis para optar al grado de Licenciatura en Psicología. Universidad de Costa Rica. San José, Costa Rica. 